\title{
Análisis factorial confirmatorio de la escala valencia de actitudes y creencias hacia la hipnosis (versión cliente revisada), en una muestra española
}

\author{
Antonio Capafons*, Jesús M. Suárez-Rodríguez y Mª Luisa Selma
}

Universitat de València, España

\begin{abstract}
Resumen: Diversas aproximaciones a la hipnosis han destacado la relevancia de las actitudes y creencias sobre ella para la promoción de las respuestas hipnóticas, para predecir la eficacia de las intervenciones que incluyen a la hipnosis, y para evitar iatrogenia en los usuarios que la reciben. El objetivo de este trabajo es investigar la nueva variación en castellano de La Escala de Valencia de Actitudes y Creencias hacia la Hipnosis versión Cliente. Se ha analizado su estructura factorial y propiedades psicométricas con metodología confirmatoria, usando una muestra exclusivamente española, para observar si se confirman los resultados de anteriores investigaciones exploratorias y confirmatorias realizadas con muestras de diferentes países. Se ha usado una muestra de 1.678 estudiantes universitarios españoles. Los resultados muestran una estructura de 8 factores (Miedo, Memoria, Ayuda, Control, Colaboración, Interés, Mágica y Marginal) y obtiene índices adecuados de consistencia interna y fiabilidad test-retest, resultados similares a los encontrados en análisis factoriales exploratorios y confirmatorios con muestras de diferentes países.

Palabras clave: Hipnosis; actitudes; evaluación; escala; análisis factorial
\end{abstract} confirmatorio; EVACH-C.
Title: Confirmatory factor analysis of the valencia scale of attitudes and beliefs toward hypnosis (revised client version), with a spanish sample.

Abstract: Several approaches to hypnosis have highlighted the importance of attitudes and beliefs on it to promote hypnotic responses, their power to predict the efficacy of interventions that include hypnosis, as well as to generate or prevent iatrogenic reactions in patients. The goal of this study is to investigate the new variation of the Valencia Scale of Attitudes and Beliefs toward Hypnosis Client version, written in Castilian. The scale was analyzed using confirmatory methodology to obtain its factor structure and psychometric properties, in order to confirm the results of previous exploratory and confirmatory research, with samples from different countries. A sample of 1.678 Spanish university students was used this time. Data show an eight factors structure (Fear, Memory, Help, Control, Collaboration, Interest, Magic and Marginal), and an appropriate internal consistency and test-retest reliability. Results are similar to those found in previous exploratory and confirmatory factorial analysis conducted in different countries.

Key words: Hypnosis; attitudes; assessment; scale; confirmatory factor analysis; VSABH-C.

\section{Introducción}

La hipnosis ha demostrado su utilidad como coadyuvante de otros procedimientos terapéuticos en el tratamiento de numerosas condiciones médicas y psicológicas, tanto en adultos como en niños, y particularmente para el tratamiento del dolor agudo y crómico (Bragado, 2009; Crawford, Knebel, Vendemia, Horton \& Lamas, 1999; Mendoza y Capafons, 2009; Orgilés, Méndez \& Espada, 2009; Palanca Sánchez et al., 2011; Truyols-Taberner, Pérez-Pareja, Palmer-Pol \& Sesé-Abad, 2008).

Antes de iniciar una intervención clínica que use los procedimientos hipnóticos como coadyuvantes es muy importante que se planteen dos objetivos: en primer lugar, evaluar las creencias y actitudes que tengan los usuarios hacia la hipnosis, y, en segundo lugar, ofrecer información científica dirigida a eliminar los posibles prejuicios y concepciones erróneas (Capafons, 2012; Capafons, Lamas \& Lopes-Pires, 2008; Spanos, Brett, Menary \& Cross, 1987).

Las razones para evaluar las creencias y actitudes de los clientes se derivan parcialmente de los dos objetivos mencionados, y se relacionan con el establecimiento del rapport y la relación terapéutica, especialmente en el caso de intervenciones clínicas. Unas actitudes positivas y adecuadas basadas en información correcta que se asocien a unas expectativas razonables sobre lo esperable del uso de la hipnosis, redundarán en una disminución de los miedos y de las expectativas exageradas sobre lo que se puede esperar de una intervención con hipnosis. Con ello se favorece la activación de

* Dirección para correspondencia [Correspondence address]:

Antonio Capafons. Facultat de Psicologia. Avda. Blasco Ibáñez, 21. 46010, Valencia (España).E-mail: antonio.capafons@uv.es las respuestas a las sugestiones hipnóticas y la adhesión a la intervención (Capafons, 2012; Hawkins \& Bartsch, 2000; Spanos et al., 1987). De este modo, se estaría contrarrestando la deleznable imagen de la hipnosis de espectáculo (Echterling \& Whalen, 1995) que tienen muchas personas.

Por otro lado, se estaría fomentando elementos como el priming, la conducta planificada y las percepciones del usuario de una manera positiva.

Todo ello ayuda a incrementar los efectos de la intervención, efecto placebo incluido (Sliwinskiand \& Elkins, 2013).

Otro aspecto importante ligado al primer objetivo es el de la evaluación de las actitudes y creencias, pues las actitudes positivas hacia la hipnosis al inicio del tratamiento predicen mejor el cambio terapéutico que el nivel de sugestionabilidad hipnótica (Schoenberger, Kirsch, Gearan, Montgomery \& Pastyrnak, 1997). Este último no suele predecir bien el resultado terapéutico cuando se usan procedimientos hipnóticos, particularmente en el campo del dolor (Jensen, 2011).

Finalmente, las creencias inadecuadas sobre la hipnosis del terapeuta y/o del paciente, son los únicos factores de riesgo demostrados que pueden generar iatrogenia, particularmente en el ámbito clínico (por ejemplo, creación de falsos recuerdos, o aplicar la hipnosis como única intervención en dolencias y problemas médicos o psicológicos).

La erradicación de ideas como que la hipnosis es en sí misma una terapia potente y que no exige esfuerzo al usuario, o que favorece la hipermnesia por encima de otros métodos al uso para mejorar la precisión y cantidad del recuerdo, o que la persona hipnotizada siempre recuerda y dice la verdad, se considera imprescindible para 
una intervención eficaz y segura de la hipnosis (Capafons, 2012; Capafons \& Mazzoni, 2005).

Por todo ello, evaluar y analizar las creencias y actitudes hacia la hipnosis tiene una larga tradición en este campo (Mendoza, Capafons y Flores, 2009). No obstante esta tradición había decaído con los años, a excepción de los estudios iniciados en España y asociados al Modelo de Valencia de Hipnosis Despierta (Alarcón y Capafons, 2011; Capafons, 1999; Capafons y Mendoza, 2010). Dentro de este modelo surgió la Escala de Valencia de Actitudes y Creencias hacia la Hipnosis versión Cliente (EVACH-C) (Capafons, Alarcón, Cabañas y Espejo, 2003; Capafons, Cabañas, Espejo y Cardeña, 2004), siendo una escala genuinamente española y redactada en castellano.

Una parte de los ítems de la EVACH-C se basaba en varios cuestionarios que evalúan actitudes hacia la hipnosis (Eimer y Freeman, 1998; Keller, 1996; McConkey, 1986; McConkey y Jupp, 1985-86; Nickisson, 1997; Spanos et al., 1987), además de incluir elementos originales no tomados de ningún otro instrumento. La escala recogía los principales mitos resumidos por Capafons $(1998,2009)$, y de los que se habla habitualmente en la literatura científica sobre la hipnosis.

Comenzó siendo un instrumento con 28 ítems y un formato de respuesta tipo likert desde 1 (desacuerdo) a 5 (completamente de acuerdo), tomado de la escala de Keller (1996), en el que 2 ya indicaba acuerdo. Esta primera versión de la EVACH-C fue analizada con metodología factorial exploratoria en un estudio realizado por Capafons et al. (2003) en el que se obtuvieron un total de seis factores: Autómata, Ayuda, Control Personal, Interés, Solución Mágica y Colaboración. La consistencia interna de la escala mostró ser satisfactoria, aunque las correlaciones testretest de los factores Solución Mágica y Colaboración necesitaban ser mejoradas $\left(r_{\mathrm{xy}}=0.54, p<.01 ; r_{\mathrm{xy}}=0.39, p<\right.$ .01 , respectivamente). No obstante, la escala fue sensible a los cambios en creencias y actitudes hacia la hipnosis provocados por un conjunto de intervenciones destinadas a modificarlas (Capafons et al., 2005; Capafons et al., 2006). Debido a ello, y porque la estructura factorial exploratoria era satisfactoria al reflejar los principales mitos y creencias erróneas hacia la hipnosis, se estudió si se mantenía en un análisis factorial confirmatorio. La estructura factorial obtenida con el análisis factorial confirmatorio replicó a la obtenida en el estudio exploratorio anterior (Capafons et al., 2004).

No obstante, la redacción de algunos ítems se mostró inadecuada, así como el formato de respuesta, ya que, como hemos indicado, una puntuación de 2 indicaba acuerdo. Por ello, se decidió modificarla añadiendo varios ítems que muestrearan algunas creencias que se habían obviado en esa versión, y que la literatura de la época indicaba que eran altamente iatrogénicas, como la supuesta capacidad de la hipnosis para generar hipermnesia y exactitud en el recuerdo. Así surgió la versión modificada de la EVACH-C. Esta variación consta de 37 ítems, con formato de respues- ta tipo likert de seis puntos indicando el 1 estar completamente en desacuerdo, y el 6 completamente de acuerdo, sin punto medio de indiferencia.

Carvalho et al. (2007) analizaron con metodología factorial exploratoria las propiedades psicométricas y la estructura factorial de la nueva versión de la EVACH-C con muestra portuguesa. El análisis factorial exploratorio mostró una estructura de 8 factores: Interés/Atracción (deseo de ser hipnotizado y de ser muy hipnotizable), Memoria/Mágica (las sugestiones hipnóticas permiten mejorar o solucionar un problema sin necesidad de esfuerzo por parte de la persona, y la persona hipnotizada puede acceder a recuerdos de sucesos pasados que no recuerda y que le impide mentir sobre lo que mentiría en condiciones normales), Ayuda (la hipnosis es una técnica que facilita los resultados terapéuticos y mejora las intervenciones médicas y psicológicas a las que se adjunta), Control (la persona hipnotizada mantiene el control en sus acciones y es capaz de resistir a las sugestiones si lo desea), Colaboración (se refiere al esfuerzo y la colaboración necesarios por parte de la persona hipnotizada para experimentar las sugestiones hipnóticas), Marginal (creencias relacionadas con considerar la hipnosis al margen de la investigación científica, y a la persona hipnotizada como crédula, mentalmente vulnerable, dependiente, etc.), Miedo (la hipnosis es peligrosa y la gente puede quedarse atrapada en ella) y Autómata (la persona hipnotizada actúa bajo el control del hipnotizador).

Capafons, Mendoza et al. (2008) realizaron un estudio multicultural usando esta misma versión de la EVACH-C en castellano y traducciones al portugués, rumano e inglés. La muestra fue obtenida en España, Portugal, Estados Unidos de Norte América y Rumania. Los resultados mostraron de nuevo una solución factorial de 8 factores, con ligeras variaciones, pues desaparece Autómata, y Memoria no se asocia a Mágica, sino a estar en un estado de trance. En concreto los factores fueron: Ayuda, Control, Mágica, Interés, Colaboración, Miedo, Memoria/Trance y Marginal.

Recientemente, Green, Houts, y Capafons (2012) han estudiado las propiedades psicométricas y la estructura factorial de la EVACH-C con una muestra de 1.141 estudiantes norteamericanos. Estos autores han obtenido de nuevo una solución de 8 factores similar a lo hallado por Carvalho et al. (2007) y Capafons, Mendoza et al. (2008). Así mismo, en este estudio los factores se muestran consistentes internamente y temporalmente estables.

La EVACH-C modificada sirvió de base para otra versión: la Escala de Valencia de Actitudes y Creencias hacia la Hipnosis, versión Terapeuta (EVACH-T). Esta escala tiene el mismo formato de respuesta, misma cantidad de ítems que la versión Cliente objeto de este estudio, pero con modificaciones para poder ser respondidos desde la perspectiva de un/a terapeuta (escala que puede encontrarse en castellano en Capafons, 2012).

Capafons, Morales, Espejo, y Cabañas (2006) obtuvie- 
ron con análisis exploratorio de la EVACH-T una estructura factorial similar a EVACH-C obtenida por Carvalho et al. (2007) y por Capafons, Mendoza et al. (2008). En concreto, los factores fueron ocho: Miedo, Memoria/Trance, Ayuda, Control, Colaboración, Interés, Mágica y Marginal. Así mismo, las propiedades psicométricas fueron satisfactorias. Análisis factoriales confirmatorios posteriores con la EVACH-T replicaron estos resultados (Capafons, Espejo y Mendoza, 2008).

Por todo ello, los objetivos de este estudio son:

1. Analizar la estructura factorial de la EVACH-C en muestra española con metodología confirmatoria, partiendo de los análisis exploratorios y confirmatorios con muestras de diferentes países comentados anteriormente.

2. Establecer la fiabilidad test-retest (estabilidad temporal), y la consistencia interna obtenida con metodología confirmatoria.

Por lo tanto, esperamos replicar la estructura de 8 factores mencionada, y unos valores de test-retest y consistencia interna variables en función de los factores, pero similares a los encontrados en lo análisis exploratorios multiculturales (Capafons et al., 2008), portugueses (Carvalho et al., 2007) y factoriales estadounidenses de Green et al. (2012), así como de la EVACH-T (Capafons et al., 2008).

\section{Método}

\section{Participantes}

1678 estudiantes universitarios españoles respondieron a la escala en ambos pases de la EVACH-C (test y retest). Un $65,75 \%$ estudiaba en instituciones universitarias y educativas de la Comunidad Valenciana (Universitat d'Alacant, Universitat Jaume I, Universitat de València E. G. y Universidad Politécnica de Valencia). Un 23.76\% lo hacía en instituciones de estos ámbitos en Cataluña (Instituto Superior de Estudios Psicológicos (ISEP)), Universitat de Barcelona y Universitat Rovira i Virgili; y un $11.16 \%$ en el resto del Estado Español (Universidad de La Coruña, Universidad Nacional a Distancia, Universidad de la Laguna y Universidad de Valladolid).

El $81.6 \%$ eran mujeres y el $18.4 \%$ hombres, con un promedio de edad de 21.1 años $(D T=5.528$; rango 17-74). El 3.04\% de los participantes había sido hipnotizado previamente, el $52 \%$ había recibido información previa acerca de la hipnosis, del cual, el $29.4 \%$ indicaba que había recibido información en la universidad, el $10.5 \%$ a través de revistas científicas, el $2.7 \%$ en cursos, el $9.5 \%$ a través de otro tipo de lecturas, el $29 \%$ a través de la televisión, y el $6 \%$ a través de otro tipo de medios (por ejemplo, conversaciones con amigos, en el colegio o en el instituto, Internet, psicólogos, etc.). Un 12.9 no indicó la procedencia de su información
Tabla 1. Titulaciones donde se aplicó la EVACH-C

\begin{tabular}{lll}
\hline Titulación & Frecuencia & Porcentaje \\
\hline Licenciatura en Psicología & 1217 & 72.7 \\
Licenciatura de Psicopedagogía & 2 & 0.1 \\
Máster en Psicología Clínica & 13 & 0.8 \\
Licenciatura de Pedagogía & 136 & 8.1 \\
Diplomatura de Magisterio & 77 & 4.6 \\
Licenciatura de Derecho & 27 & 1.6 \\
Licenciatura de Sociología & 55 & 3.3 \\
Licenciatura de Periodismo & 2 & 0.1 \\
Diplomatura de Logopedia & 82 & 4.9 \\
Ingeniería en Telecomunicacio- & 0.1 & \\
nes & 1 & \\
Licenciatura de Administración & 3.6 & \\
y Dirección de Empresas-60 & & \\
Economía & 1078 & \\
Total & &
\end{tabular}

Tabla 2. Distribución de los estudiantes por cursos de licenciatura o máster.

\begin{tabular}{lll}
\hline Curso & Frecuencia & Porcentaje \\
\hline $1^{\circ}$ & 567 & 34.1 \\
$2^{\circ}$ & 498 & 29.9 \\
$3^{\circ}$ & 263 & 15.8 \\
$4^{\circ}$ & 166 & 10.0 \\
$5^{\circ}$ & 28 & 1.7 \\
Máster en Psicología Clínica & 142 & 8.5 \\
Total & 1678 & 100 \\
\hline
\end{tabular}

Nota. \% casos válidos, un $0.8 \%$ no responde

\section{Instrumentos}

Se usó la EVACH-C (Capafons et al., 2008), en la variación de 37 ítems ya indicada, escrita en castellano de España (ya que existe una versión para Cuba). Además de esos 37 ítems, la escala incluye unas preguntas previas que recogen información acerca de los datos de identificación, sexo, del nivel de formación universitaria, si han sido hipnotizados previamente, conocimientos sobre hipnosis y medios a través de los que se han obtenido, etc. Puede encontrase la EVACH-C en el Apéndice.

\section{Procedimiento}

Los pases se realizaron en grupos por la tercera autora de este trabajo, por otro profesorado de la Universitat de València, y por profesorado del resto de las universidades e institutos mencionados. A cada persona que debía aplicar la escala se le enviaba una carta con instrucciones muy específicas acerca de cómo presentarla, qué se debía verbalizar, además de proporcionar aclaraciones, si eran necesarias, a través del teléfono y otros medios disponibles.

A los participantes se les decía que se trataba de un estudio para validar una escala que evaluaba las creencias y actitudes acerca de la hipnosis. Se enfatizó que no existían respuestas buenas o malas y se pidió que respondieran a todos los ítems, señalando la alternativa que mejor reflejara su opinión sobre cada cuestión concreta. Asimismo, se les decía que las respuestas eran anónimas, garantizándose la confi- 
dencialidad de la identidad de los participantes, pero que escribieran las 4 últimas cifras y letra del DNI para poder organizar los ejemplares). Finalmente, se les comunicaba que la participación en el estudio era voluntaria sin contraprestación económica ni académica alguna.

Sobre el pase del retest, realizado un mes más tarde, quienes aplicaban la prueba debían decir a los participantes que se hacía un segundo pase porque se habían perdido parte de los datos del primero, y que se centraran en lo que pensaban en ese momento, no en lo que pudieran haber respondido en el pase anterior.

Tabla 3. Estadísticos descriptivos y prueba de normalidad univariada en los

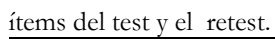

\begin{tabular}{|c|c|c|c|c|}
\hline \multirow[b]{2}{*}{ Variable } & \multirow[b]{2}{*}{ Media } & \multirow[b]{2}{*}{$D T$} & \multicolumn{2}{|c|}{ Asimetría y Curtosis } \\
\hline & & & $\chi^{2}$ & Probabilidad \\
\hline Ítem 1 & 4.18 & 1.00 & 120.58 & .000 \\
\hline Ítem 2 & 4.95 & 0.98 & 170.91 & .000 \\
\hline Ítem 3 & 3.86 & 1.32 & 46.03 & .000 \\
\hline Ítem 4 & 3.12 & 1.58 & 82.83 & .000 \\
\hline Ítem 5 & 2.70 & 1.24 & 50.07 & .000 \\
\hline Ítem 6 & 2.33 & 1.25 & 99.62 & .000 \\
\hline Ítem 7 & 3.40 & 1.38 & 32.29 & .000 \\
\hline Ítem 8 & 4.24 & 1.13 & 83.25 & .000 \\
\hline Ítem 9 & 2.14 & 1.13 & 302.12 & .000 \\
\hline Ítem 10 & 3.89 & 1.12 & 59.28 & .000 \\
\hline Ítem 11 & 2.91 & 1.20 & 30.38 & .000 \\
\hline Ítem 12 & 4.29 & 1.07 & 144.50 & .000 \\
\hline Ítem 13 & 5.03 & 1.00 & 230.43 & .000 \\
\hline Ítem14 & 2.76 & 1.24 & 68.89 & .000 \\
\hline Ítem 15 & 2.79 & 1.21 & 62.49 & .000 \\
\hline Ítem 16 & 3.25 & 1.10 & 4.950 & .084 \\
\hline Ítem 17 & 3.20 & 1.06 & 14.28 & .001 \\
\hline Ítem 18 & 3.14 & 1.49 & 52.62 & .000 \\
\hline Ítem 19 & 3.50 & 1.28 & 17.61 & .000 \\
\hline Ítem 20 & 3.32 & 1.28 & 12.77 & .002 \\
\hline Ítem 21 & 3.04 & 1.12 & 27.24 & .000 \\
\hline Ítem 22 & 3.51 & 1.31 & 22.28 & .000 \\
\hline Ítem 23 & 3.92 & 1.02 & 107.33 & .000 \\
\hline Ítem 24 & 3.64 & 1.24 & 2.83 & .243 \\
\hline Ítem 25 & 3.03 & 1.18 & 39.84 & .000 \\
\hline Ítem 26 & 3.68 & 1.68 & 92.96 & .000 \\
\hline Ítem 27 & 3.85 & 1.56 & 75.70 & .000 \\
\hline Ítem 28 & 2.82 & 1.44 & 63.41 & .000 \\
\hline Ítem 29 & 2.76 & 1.13 & 32.08 & .000 \\
\hline Ítem 30 & 3.27 & 1.26 & 2.05 & .358 \\
\hline Ítem 31 & 3.55 & 1.26 & 8.98 & .011 \\
\hline Ítem 32 & 3.70 & 1.27 & 32.26 & .000 \\
\hline Ítem 33 & 4.13 & 1.07 & 108.13 & .000 \\
\hline Ítem 34 & 3.19 & 1.21 & 3.54 & .170 \\
\hline Ítem 35 & 2.85 & 1.27 & 26.02 & .000 \\
\hline Ítem 36 & 3.04 & 1.12 & 6.84 & .033 \\
\hline Ítem 37 & 4.10 & 1.17 & 59.94 & .000 \\
\hline Ítemr 1 & 4.17 & 0.90 & 67.81 & .000 \\
\hline Ítemr 2 & 4.78 & 0.79 & 103.97 & .000 \\
\hline Ítemr 3 & 3.78 & 0.97 & 108.92 & .000 \\
\hline Ítemr 4 & 3.06 & 1.22 & 27.16 & .000 \\
\hline Ítemr 5 & 2.60 & 0.98 & 118.94 & .000 \\
\hline Ítemr 6 & 2.33 & 1.02 & 77.67 & .000 \\
\hline Ítemr 7 & 3.08 & 1.17 & 6.83 & .033 \\
\hline Ítemr 8 & 4.27 & 0.86 & 61.16 & .000 \\
\hline
\end{tabular}

\begin{tabular}{|c|c|c|c|c|}
\hline Itemr 9 & 2.28 & 0.83 & 342.03 & .000 \\
\hline Ítemr 10 & 3.40 & 0.97 & 48.41 & .000 \\
\hline Ítemr 11 & 2.96 & 0.95 & 45.17 & .000 \\
\hline Ítemr 12 & 4.29 & 0.94 & 72.09 & .000 \\
\hline Ítemr 13 & 4.87 & 0.82 & 222.53 & .000 \\
\hline Ítemr 14 & 3.01 & 1.06 & 74.19 & .000 \\
\hline Ítemr 15 & 3.02 & 1.01 & 137.95 & .000 \\
\hline Ítemr 16 & 3.42 & 0.92 & 53.51 & .000 \\
\hline Ítemr 17 & 3.32 & 0.88 & 151.74 & .000 \\
\hline Ítemr 18 & 2.96 & 1.22 & 23.16 & .000 \\
\hline Ítemr 19 & 3.27 & 1.05 & 12.58 & .002 \\
\hline Ítemr 20 & 3.14 & 1.12 & 0.44 & .803 \\
\hline Ítemr 21 & 3.16 & 0.84 & 357.37 & .000 \\
\hline Ítemr 22 & 3.29 & 1.10 & 14.65 & .001 \\
\hline Ítemr 23 & 4.06 & 0.87 & 123.50 & .000 \\
\hline Ítemr 24 & 3.75 & 0.95 & 43.13 & .000 \\
\hline Ítemr 25 & 3.18 & 1.00 & 94.88 & .000 \\
\hline Ítemr 26 & 3.59 & 1.45 & 51.24 & .000 \\
\hline Ítemr 27 & 3.74 & 1.41 & 44.96 & .000 \\
\hline Ítemr 28 & 2.88 & 1.25 & 31.64 & .000 \\
\hline Ítemr 29 & 2.81 & 0.86 & 152.83 & .000 \\
\hline Ítemr 30 & 3.25 & 0.95 & 57.75 & .000 \\
\hline Ítemr 31 & 3.39 & 1.01 & 21.73 & .000 \\
\hline Ítemr 32 & 3.52 & 1.01 & 28.61 & .000 \\
\hline Ítemr 33 & 4.03 & 0.84 & 592.32 & .000 \\
\hline Ítemr 34 & 3.13 & 0.96 & 53.60 & .000 \\
\hline Ítemr 35 & 2.71 & 1.00 & 16.91 & .000 \\
\hline Ítemr 36 & 2.98 & 0.91 & 120.40 & .000 \\
\hline Ítemr 37 & 4.23 & 1.00 & 34.89 & .000 \\
\hline
\end{tabular}

Mediante el programa SPSS 19.0 obtuvimos estadísticos descriptivos del test y del retest. Además, hicimos análisis correlacionales entre los elementos de la prueba en el primer momento de medida (test) sobre el que se ha contrastado el modelo de medida. Así mismo,_realizamos un Análisis Factorial Confirmatorio $(A F C$ ) mediante el programa LISREL 8.80 (Jöreskog, Sörbom, Du Toit y Du Toit, 1999). En cuanto a las estimaciones del modelo para confirmar la dimensionalidad de la escala, las hemos realizado mediante el procedimiento de Máxima Verosimilitud Robusto (RML), dada la no normalidad de las dimensiones que se utilizan (Tabla 3).

Para la evaluación del ajuste del modelo se ha utilizado la $\chi^{2}$ ajustada según la propuesta de Satorra-Bentler, debido al procedimiento elegido (Satorra y Bentler, 1994). Asimismo utilizamos otros indicadores acordes a las recomendaciones de Byrne (2006), Hair, Black, Babib, y Anderson (2010) y Kline (2005). A partir de estas recomendaciones, hemos seleccionado el error cuadrático medio de aproximación (RMSE $A$ ), en el que se considera un buen ajuste un valor menor de 0.05 , considerado junto con su intervalo de confianza al 90\%. Además, presentamos los índices de ajuste incremental, Índice de Ajuste Comparativo (CFI) e Índice No Normalizado de Ajuste (NNFI), en los que se considera un buen ajuste valores iguales o superiores a 0.95 . También incluimos el Residuo Cuadrático Medio Estandarizado 
(SRMR), cuyos valores iguales o menores a 0.08 indican un ajuste aceptable.

Para valorar la fiabilidad de las dimensiones hemos utilizado el indicador propuesto por Raykov $(2001,2004)$ en el caso del modelo de Análisis Factorial Confirmatorio ( $A F C$, $\mathrm{y}$ hemos añadido información respecto al coeficiente alfa de Cronbach (1951).

Finalmente, hemos comprobado la estabilidad de las dimensiones mediante fiabilidad test-retest a partir de $A F C$.

\section{Resultados}

En cuanto a los estadísticos descriptivos para cada ítem, tanto del test como del retest se encontraron los resultados mostrados en la tabla 3. Se aprecia una razonable dispersión en los elementos de la prueba, tanto en el test como en el retest, dada la fuerte no normalidad generalizada en ambos pases, ya comentada en la elección del procedimiento de estimación del modelo.

En la tabla 4 se muestran los resultados correlacionales entre los elementos de la prueba en el primer momento de medida (test) sobre el que se ha contrastado el modelo de medida.

Tabla 4. Matriz de correlaciones entre los ítems del test.

\begin{tabular}{|c|c|c|c|c|c|c|c|c|c|c|c|c|}
\hline Ítem 4 & $\begin{array}{l}\text { Item } 4 \\
1.00\end{array}$ & Ítem 7 & Ítem16 & Ítem18 & Ítem19 & Ítem 20 & Ítem 3 & Ítem 30 & Ítem 31 & Ítem 32 & Ítem 33 & Ítem 1 \\
\hline Ítem 7 & .25 & 1.00 & & & & & & & & & & \\
\hline Ítem 16 & -.32 & -.24 & 1.00 & & & & & & & & & \\
\hline Ítem 18 & .57 & .35 & -.32 & 1.00 & & & & & & & & \\
\hline Ítem 19 & .36 & .45 & -.30 & .48 & 1.00 & & & & & & & \\
\hline Ítem 20 & .52 & .35 & -.54 & .59 & .53 & 1.00 & & & & & & \\
\hline Ítem 3 & .09 & .19 & .03 & .14 & .13 & .07 & 1.00 & & & & & \\
\hline Ítem 30 & .02 & .19 & .12 & .09 & .13 & .00 & .14 & 1.00 & & & & \\
\hline Ítem 31 & .14 & .35 & -.02 & .21 & .28 & .13 & .20 & .49 & 1.00 & & & \\
\hline Ítem 32 & .07 & .30 & .01 & .14 & .20 & .08 & .23 & .50 & .57 & 1.00 & & \\
\hline Ítem 33 & .09 & .26 & -.07 & .14 & .22 & .13 & .26 & .19 & .27 & .32 & 1.00 & \\
\hline Ítem 1 & -.11 & .00 & .21 & -.07 & -.02 & -.17 & .19 & .19 & .16 & .22 & .11 & 1.00 \\
\hline Ítem 10 & -.16 & -.03 & .29 & -.08 & -.04 & -.22 & .07 & .19 & .09 & .17 & .09 & .49 \\
\hline Ítem 12 & -.15 & -.01 & .30 & -.08 & .00 & -.19 & .11 & .23 & .14 & .23 & .20 & .51 \\
\hline Ítem 17 & -.15 & -.14 & .43 & -.11 & -.22 & -.26 & .07 & .11 & -.03 & .03 & .00 & .22 \\
\hline Ítem 23 & -.15 & -.04 & .32 & -.06 & -.05 & -.18 & .07 & .23 & .10 & .18 & .13 & .43 \\
\hline Ítem 29 & -.06 & .08 & .21 & .04 & .05 & -.08 & .14 & .29 & .15 & .20 & .10 & .29 \\
\hline Ítem 37 & -.17 & -.04 & .30 & -.07 & -.06 & -.20 & .08 & .22 & .14 & .23 & .17 & .47 \\
\hline Ítem 14 & -.15 & -.36 & .30 & -.22 & -.32 & -.25 & -.13 & -.13 & -.30 & -.28 & -.21 & -.02 \\
\hline Ítem 15 & -.21 & -.48 & .39 & -.23 & -.41 & -.30 & -.16 & -.09 & -.32 & -.25 & -.18 & .06 \\
\hline Ítem 21 & .00 & -.12 & .13 & -.03 & -.07 & -.01 & -.03 & .01 & -.07 & -.03 & -.08 & -.06 \\
\hline Ítem 22 & .27 & .56 & -.34 & .38 & .48 & .43 & .15 & .13 & .31 & .27 & .20 & -.01 \\
\hline Ítem 24 & -.13 & -.22 & .24 & -.15 & -.18 & -.16 & -.08 & -.04 & -.17 & -.10 & .02 & .09 \\
\hline Ítem 25 & -.22 & -.47 & .35 & -.26 & -.39 & -.30 & -.18 & -.12 & -.32 & -.26 & -.21 & .05 \\
\hline Ítem 2 & -.08 & -.02 & .15 & -.05 & -.01 & -.09 & .16 & .11 & .09 & .15 & .14 & .40 \\
\hline Ítem 8 & .00 & -.10 & .04 & -.01 & -.01 & -.04 & .07 & .00 & -.06 & .01 & .02 & .17 \\
\hline Ítem 13 & -.03 & -.03 & .08 & -.03 & .01 & -.07 & .13 & .03 & .03 & .09 & .13 & .24 \\
\hline Ítem 26 & -.45 & -.09 & .28 & -.26 & -.15 & -.35 & .08 & .13 & .03 & .17 & .11 & .38 \\
\hline Ítem 27 & -.47 & -.12 & .29 & -.31 & -.17 & -.37 & .06 & .13 & .04 & .17 & .08 & .36 \\
\hline Ítem 28 & -.31 & -.02 & .28 & -.16 & -.10 & -.28 & .09 & .21 & .08 & .18 & .10 & .32 \\
\hline Ítem 5 & .14 & .32 & -.01 & .19 & .18 & .13 & .11 & .13 & .19 & .16 & .11 & -.04 \\
\hline Ítem 6 & .14 & .39 & -.01 & .25 & .22 & .13 & .22 & .28 & .33 & .26 & .13 & .12 \\
\hline Ítem 9 & .05 & .19 & .09 & .18 & .11 & .04 & .20 & .25 & .24 & .20 & .10 & .17 \\
\hline Ítem 34 & .14 & .16 & -.13 & .15 & .10 & .19 & .08 & -.01 & .08 & .04 & .13 & -.17 \\
\hline Ítem 35 & .15 & .20 & -.19 & .18 & .21 & .23 & .03 & .03 & .06 & .06 & .05 & -.18 \\
\hline Ítem 36 & .16 & .22 & -.17 & .19 & .23 & .25 & .13 & .15 & .19 & .13 & .20 & -.05 \\
\hline
\end{tabular}


Tabla 4. Matriz de correlaciones entre los ítems del test (continuada)

\begin{tabular}{|c|c|c|c|c|c|c|c|c|c|c|c|c|}
\hline & item10 & item12 & item17 & item 23 & item29 & item 37 & item14 & item15 & item 21 & item 22 & item24 & item 25 \\
\hline Item 10 & 1.00 & & & & & & & & & & & \\
\hline Item12 & .61 & 1.00 & & & & & & & & & & \\
\hline Item17 & .33 & .30 & 1.00 & & & & & & & & & \\
\hline Item 23 & .58 & .58 & .36 & 1.00 & & & & & & & & \\
\hline Item29 & .33 & .29 & .34 & .36 & 1.00 & & & & & & & \\
\hline Item 37 & .57 & .65 & .33 & .60 & .29 & 1.00 & & & & & & \\
\hline Item14 & .06 & .01 & .27 & .11 & .06 & .05 & 1.00 & & & & & \\
\hline Item15 & .14 & .08 & .35 & .13 & .13 & .11 & .65 & 1.00 & & & & \\
\hline Item 21 & -.03 & -.05 & .16 & .03 & .08 & .00 & .27 & .25 & 1.00 & & & \\
\hline Item 22 & -.04 & -.04 & -.20 & -.08 & .05 & -.08 & -.38 & -.50 & -.11 & 1.00 & & \\
\hline Item 24 & .12 & .11 & .20 & .14 & .03 & .12 & .27 & .35 & .11 & -.24 & 1.00 & \\
\hline Item 25 & .10 & .06 & .33 & .15 & .09 & .11 & .53 & .65 & .29 & -.52 & .35 & 1.00 \\
\hline Item2 & .32 & .33 & .17 & .27 & .14 & .32 & -.01 & .05 & .02 & -.01 & .14 & .08 \\
\hline Item8 & .18 & .21 & .12 & .15 & .01 & .14 & .07 & .11 & .08 & -.08 & .13 & .13 \\
\hline Item13 & .24 & .34 & .11 & .22 & .02 & .23 & .02 & .06 & .01 & -.03 & .14 & .05 \\
\hline Item 26 & .36 & .36 & .24 & .31 & .32 & .34 & .09 & .14 & .00 & -.15 & .06 & .16 \\
\hline Item 27 & .36 & .36 & .23 & .30 & .30 & .32 & .10 & .15 & .02 & -.16 & .07 & .16 \\
\hline Item 28 & .34 & .28 & .26 & .27 & .43 & .28 & .08 & .17 & .04 & -.07 & .05 & .12 \\
\hline Item5 & -.03 & -.07 & -.02 & -.05 & .15 & -.03 & -.09 & -.12 & .04 & .26 & -.10 & -.16 \\
\hline Item6 & .07 & .09 & .06 & .10 & .25 & .10 & -.15 & -.18 & .04 & .28 & -.16 & -.20 \\
\hline Item9 & .18 & .13 & .18 & .16 & .29 & .19 & -.05 & -.02 & .07 & .16 & -.11 & -.09 \\
\hline Item 34 & -.23 & -.19 & -.11 & -.18 & -.03 & -.19 & -.05 & -.07 & .07 & .13 & -.02 & -.11 \\
\hline Item 35 & -.25 & -.20 & -.13 & -.16 & .00 & -.15 & -.05 & -.11 & .09 & .21 & -.02 & -.11 \\
\hline Item 36 & -.10 & -.05 & -.08 & -.03 & .08 & -.03 & -.11 & -.18 & -.01 & .27 & -.04 & -.16 \\
\hline
\end{tabular}

Tabla 4. Matriz de correlaciones entre los ítems del test (continuada)

\begin{tabular}{|c|c|c|c|c|c|c|c|c|c|c|c|c|}
\hline & item2 & item8 & item 13 & item 26 & item 27 & item 28 & item5 & item6 & item9 & item34 & item 35 & item36 \\
\hline Item2 & 1.00 & & & & & & & & & & & \\
\hline Item8 & .39 & 1.00 & & & & & & & & & & \\
\hline Item13 & .49 & .44 & 1.00 & & & & & & & & & \\
\hline Item26 & .26 & .08 & .15 & 1.00 & & & & & & & & \\
\hline Item 27 & .27 & .13 & .17 & .90 & 1.00 & & & & & & & \\
\hline Item 28 & .18 & .03 & .06 & .72 & .69 & 1.00 & & & & & & \\
\hline Item5 & -.15 & -.29 & -.23 & -.07 & -.08 & .05 & 1.00 & & & & & \\
\hline Item6 & -.03 & -.07 & -.15 & .02 & .00 & .13 & .44 & 1.00 & & & & \\
\hline Item9 & -.01 & -.03 & -.09 & .10 & .05 & .22 & .23 & .44 & 1.00 & & & \\
\hline Item34 & -.10 & -.09 & -.09 & -.15 & -.15 & -.08 & .16 & .13 & .05 & 1.00 & & \\
\hline Item 35 & -.15 & -.09 & -.17 & -.16 & -.21 & -.14 & .20 & .18 & .12 & .29 & 1.00 & \\
\hline Item36 & -.05 & -.07 & -.11 & -.11 & -.14 & -.02 & .14 & .17 & .09 & .15 & .39 & 1.00 \\
\hline
\end{tabular}

En la tabla 5 se muestran los valores obtenidos en este estudio para los indicadores de ajuste seleccionados, junto a los que se han obtenido en otros estudios ya mencionados. Los indicadores de ajuste muestran un buen resultado en general y aceptable en el caso de SRMR. Así, se ha obtenido para el modelo un valor $\chi^{2}$ s-B, como la mejor opción para la situación de no normalidad comentada.

Aunque su significación es inferior a .05, como exponen Hair et al. (2010), éste es un indicador que se ve puede ver afectado por el número de participantes y la complejidad del modelo. De hecho, el indicador basado en $\chi^{2}$ o la versión normalizada con los grados de libertad han dejado de ser desde hace algunos años considerados como un criterio cla- ve para valorar el ajuste del modelo (Hair et al., 2010; Hooper, Coughlan y Mullen, 2008; Kline, 2005). No obstante, seguimos la recomendación generalmente aceptada de informar sobre el mismo. Entre los indicadores orientados a residuos se aprecia un buen ajuste en el RMSE $A$, con un intervalo de confianza al $90 \%$ de rango muy estrecho y situado entre .049 y .052, y un ajuste satisfactorio en el caso del SRMR, al encontrarse por debajo de .08 .

Los indicadores de ajuste relativo NNFI y CFI muestran un ajuste excelente. Otro tanto se puede decir del modelo de verificación test-retest que demuestra un patrón similar, salvo en lo referente al indicador de residuos $S R M R$ que resulta menos satisfactorio. 
Tabla 5. Índices de ajuste del Modelo del $A F C$ y otros modelos ya publicados

\begin{tabular}{llllll}
\hline Indicador & Capafons et al. (2008) & Carvalho et al. (2007) & Green et al. 2012) & Modelo actual & Test-retest \\
\hline RMSEA & .069 & .061 & .058 & .050 & .056 \\
NNFI & .933 & .947 & .954 & .95 & .96 \\
SRMR & .131 & .104 & .104 & .078 & .098 \\
CFI & .939 & .950 & .958 & .95 & .96 \\
$\chi^{2}$ ajustada de & 2009.02 & 1714.07 & 1500.10 & 2972.02 & 14940.35 \\
Satorra-Bentler & 602 & 601 & 566 & 566 & 2364 \\
Grados de libertad & 602 & &
\end{tabular}

La estructura factorial encontrada se muestra en la tabla 6 , en la que se pone a prueba el modelo $A F C$ con los 8 factores predichos por la construcción de la misma y las experiencias anteriores: Miedo, Memoria, Ayuda, Control, Colaboración, Interés, Mágica y Marginal. De ella, se ha eliminado el ítem 11 ("La persona hipnotizada es pasiva"), debido a su comportamiento poco consistente. Este elemento ha mostrado un comportamiento insatisfactorio en los análisis exploratorios previos, cuestión que ya había aparecido en al- gún estudio anterior, por lo que se ha optado por eliminarlo de la estructura a la espera de considerar otras alternativas en el futuro.

En la tabla 7 se ofrecen las correlaciones entre los 8 factores. Las correlaciones son todas significativas al nivel de .05 y una buena parte al nivel de .01 , lo que muestra una conexión entre las diferentes dimensiones de creencias y actitudes sobre la hipnosis como ámbito general de referencia.

Tabla 6. Saturaciones de los ítems en los factores en la muestra española a partir del modelo $A F C$.

\begin{tabular}{|c|c|c|c|c|c|c|c|c|c|}
\hline \multicolumn{10}{|c|}{ ÍtemFactor } \\
\hline & Factor & 1:Factor & 2:Factor & 3:Factor & 4:Factor & 5:Factor & 6:Factor & 7:Factor & 8: \\
\hline & Miedo & Memoria & Ayuda & Control & Colaboración & Interés & Mágica & Marginal & \\
\hline 1 & & & .60 & & & & & & \\
\hline 2 & & & & & .65 & & & & \\
\hline 3 & & .32 & & & & & & & \\
\hline 4 & .61 & & & & & & & & \\
\hline 5 & & & & & & & .51 & & \\
\hline 6 & & & & & & & .73 & & \\
\hline 7 & .53 & & & & & & & & \\
\hline 8 & & & & & .51 & & & & \\
\hline 9 & & & & & & & .48 & & \\
\hline 10 & & & .73 & & & & & & \\
\hline 11 & & & & & & & & & \\
\hline 12 & & & .76 & & & & & & \\
\hline 13 & & & & & .64 & & & & \\
\hline 14 & & & & .68 & & & & & \\
\hline 15 & & & & .79 & & & & & \\
\hline 16 & -.53 & & & & & & & & \\
\hline 17 & & & .43 & & & & & & \\
\hline 18 & .68 & & & & & & & & \\
\hline 19 & .65 & & & & & & & & \\
\hline 20 & .76 & & & & & & & & \\
\hline 21 & & & & .28 & & & & & \\
\hline 22 & & & & -.62 & & & & & \\
\hline 23 & & & .71 & & & & & & \\
\hline 24 & & & & .40 & & & & & \\
\hline 25 & & & & .75 & & & & & \\
\hline 26 & & & & & & .93 & & & \\
\hline 27 & & & & & & .92 & & & \\
\hline 28 & & & & & & .70 & & & \\
\hline 29 & & & .42 & & & & & & \\
\hline 30 & & .59 & & & & & & & \\
\hline 31 & & .71 & & & & & & & \\
\hline 32 & & .74 & & & & & & & \\
\hline 33 & & .40 & & & & & & & \\
\hline 34 & & & & & & & & .41 & \\
\hline 35 & & & & & & & & .63 & \\
\hline 36 & & & & & & & & .50 & \\
\hline 37 & & & .74 & & & & & & \\
\hline
\end{tabular}

Nota. La redacción de los ítems se encuentra en el Apéndice. 
Tabla 7. Correlaciones entre los factores en el Modelo del AFC.

\begin{tabular}{|c|c|c|c|c|c|c|c|}
\hline 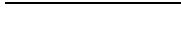 & Miedo & Memoria & Ayuda & Control & Colabor. & Interés & Mágica \\
\hline \multicolumn{8}{|l|}{ MIEDO } \\
\hline MEMORIA & .28 & & & & & & \\
\hline AYUDA & -.27 & .34 & & & & & \\
\hline CONTROL & -.60 & -.46 & .18 & & & & \\
\hline COLABOR. & -.10 & .16 & .50 & .12 & & & \\
\hline INTERÉS & -.45 & .18 & .50 & .19 & .28 & & \\
\hline MÁGICA & .33 & .51 & .18 & -.30 & -.23 & .03 & \\
\hline MARGINAL & .48 & .21 & -.33 & -.26 & -.29 & -.28 & .38 \\
\hline
\end{tabular}

Nota. COLABOR. $=$ Colaboración

En base a los índices obtenidos mediante el modelo $A F C$, se confirma la consistencia en la muestra de universitarios españoles del modelo propuesto basado en ocho factores: Miedo, Memoria, Ayuda, Control, Colaboración, Interés, Mágica y Marginal.

La fiabilidad a partir del $A F C\left(\rho_{\mathrm{xx}}\right)$ y la consistencia interna $(\alpha)$ (tabla 8) son aceptables, oscilando entre 0.58 en Control, y 0.87 en Memoria, siendo el resto superiores a 0.60. Los coeficientes alfa muestran niveles similares con so- breestimaciones e infraestimaciones en las diferentes dimensiones respecto a $\rho_{\mathrm{xx}}$ aproximadamente equilibradas en el conjunto de las ocho dimensiones. En este caso, el valor más reducido se da en la dimensión Marginal (0.50), existiendo cuatro dimensiones por encima de 0.75 . La estabilidad de los factores resulta excelente, situándose en la gran mayoría de los casos por encima de 0.90, salvo en el caso de las dimensiones Miedo (0.87) y Marginal (0.75).

Tabla 8. Fiabilidad de las dimensiones obtenidas a partir del modelo de medida estructural (Raykov), $\alpha$ de Cronbach y test re-test.

\begin{tabular}{|c|c|c|c|c|c|c|c|c|}
\hline & Miedo & Memoria & Ayuda & Control & Colaboración & Interés & Mágica & Marginal \\
\hline$\rho_{\mathrm{xx}}$ & .64 & .87 & .67 & .58 & .62 & .80 & .70 & .71 \\
\hline$\alpha$ & .79 & .67 & .82 & .76 & .63 & .88 & .58 & .50 \\
\hline TRT-AFC & .87 & .86 & .97 & .93 & .98 & .95 & .96 & .75 \\
\hline
\end{tabular}

Nota. TRT- $A F C=$ Test-retest a partir de $A F C$

Finalmente, en la tabla 9 aportamos los resultados descriptivos de los distintos factores de la escala (Media y DT) en el test y en el retest, que dan una idea general de las opiniones concretas de esta muestra. Los factores Ayuda, Control, Colaboración e Interés son factores positivos (en general, a más puntuación mejor actitud y mayor conocimiento de la investigación científica sobre la hipnosis), y el resto de factores, todos empezados curiosamente con la M (Miedo, Memoria, Mágica y Marginal) son negativos, por lo que puntuaciones elevadas, también en general, indicarían un pobre conocimiento de la hipnosis científica así como actitudes no muy adecuadas, sean o no positivas, hacia la hipnosis.

Tabla 9. Datos descriptivos de los factores de la EVACH-C en el test y en el retest.

\begin{tabular}{lllll}
\hline & Test & \multicolumn{3}{c}{ Retest } \\
\hline Miedo & Media & $\boldsymbol{D} \boldsymbol{T}$ & Media & $\boldsymbol{D T}$ \\
Memoria & 3.37 & .94 & 3.21 & .86 \\
Ayuda & 3.70 & .82 & 3.59 & .68 \\
Control & 3.76 & .74 & 3.84 & .70 \\
Colaboración & 3.12 & .81 & 3.26 & .75 \\
Interés & 3.45 & .78 & 4.64 & .68 \\
Mágica & 2.39 & .89 & 3.43 & 1.31 \\
Marginal & 3.02 & .85 & 2.40 & .74 \\
\hline Nota. DT & Desviacion th & .71
\end{tabular}

Nota. DT $=$ Desviación típica

\section{Conclusiones y discusión}

Los datos sustentan la primera predicción, pues la solución factorial alcanzada se corresponde con las soluciones factoriales de los análisis factoriales exploratorios realizados con muestras multiculturales (Capafons, Mendoza et al., 2008). Asimismo es muy similar a la solución factorial exploratoria obtenidas con la escala por Carvalho et al (2007), en la que también aparecieron los mismos 8 factores, si bien con aglutinaciones de ítems ligeramente distintas. También es semejante a la estructura factorial confirmatoria obtenida por Green et al. (2012) con muestra norteamericana, si bien algunos los ítems se redistribuyeron de una manera ligeramente diferente a lo encontrado en los estudios con muestras portuguesas y españolas, pero, en todo caso, siempre manteniendo la consistencia teórica y lógica de la ubicación de los ítems. Además es consistente con la solución factorial confirmatoria alcanzada con la versión EVACH-T (Capafons, Espejo et al., 2008).

Por lo tanto, la estructura encontrada ratifica y confirma los resultados de otras investigaciones con análisis exploratorios y confirmatorios, además de los de la escala gemela destinada a terapeutas. Y lo que no es menos importante, con muestras de, al menos, tres países. Resultados con $A F C$ en proceso de redacción obtenidos con muestras portuguesas (Capafons, Flores, Suárez \& Lopes-Pires, 2010) también están ofreciendo resultados prácticamente idénticos.

Por otro lado, en cuanto a la calidad de los resultados, la 
escala alcanza ajustes satisfactorios ligeramente mejores que en la muestra norteamericana. Su fiabilidad más robusta es la de test-retest, luego Raykov, y, por último, $\alpha$, menor que en la muestra portuguesa (metodología factorial exploratoria) y que en la muestra norteamericana.

En general, se replican también los resultados, lo que indica la robustez de la escala. Aún así, conviene indicar que los datos de los factores Control y Colaboración presentan limitaciones para decisiones individuales en cuanto a fiabilidad de las dimensiones, aunque sus niveles de estabilidad permiten avalar en las condiciones actuales la consistencia de la información que ofrecen.

En cuanto a investigaciones futuras, sería interesante seguir adaptando la escala a otros países, como nos consta que se está haciendo en Australia, Colombia, Cuba, Nueva Zelanda y Venezuela, así como que se están iniciando procesos de investigación en esta dirección en Perú y Chile. En este sentido, es importante también utilizar muestras clínicas, ya que en el presente estudio se han incluido fundamentalmente estudiantes, lo que podría sesgar los resultados. No obstante, estos mismos resultados, como se ha indicado, son similares a los obtenidos con la escala versión terapeuta (EVACH-T) y con estudiantes de otros países.

Por otro lado, se podrían comparar las puntuaciones y perfiles que se obtuvieran con la escala entre profesiones, países, etc., algo que ya se comenzó hace tiempo con resultados interesantes obtenidos en muestras cubanas usando la EVACH-C (Capafons, Espejo \& Cabañas, 2005), y con la EVACH-T en muestras españolas (Mendoza, Capafons, Espejo \& Montalvo, 2009). En la muestra de este estudio se observa que los factores positivos no alcanzan puntuaciones muy altas, siendo la mayor la de Colaboración. Es interesante observar que los factores negativos tampoco destacan por sus valores elevados, siendo el más alto el del factor Memoria. Es decir, ni grandes filias ni grandes fobias hacia la hipnosis, así como una aparente sensatez en cuanto a lo que

\section{Referencias}

Alarcón, A., \& Capafons, A. (2006). El modelo de Valencia de hipnosis despierta: ¿técnicas nuevas o técnicas innovadoras? Papeles del Psicólogo, 27, $70-78$.

Bragado, C. (2009). Funcionamiento psicosocial e intervenciones psicológicas en niños con cáncer. Psicooncología, 6, 327-341.

Byrne, B. (2006). Structural equation modeling with EQS. Basic concepts, applications, and programming ( $2^{\mathrm{a}}$ ed.). Mahawah, NJ: Lawrence Erlbaum Associates Publishers.

Capafons, A. (1998). Hipnosis clínica: una visión cognitivocomportamental. Papeles del Psicólogo, 69, 71-88.

Capafons, A. (1999). La hipnosis despierta setenta y cuatro años después. Anales de Psicología, 15, 77-78.

Capafons, A. (2009). Diez años después de "hipnosis clínica: una visión cognitivo comportamental”. En C. M. Lopes Pires \& E. Santos (Eds.), Hipnose clínica: Fundamentos e aplicações em psicologia e saúde (pp. 49-57). Viseu, Portugal: Psicossoma.

Capafons, A. (2012). Hipnosis. Segunda edición ampliada y revisada. Madrid: Síntesis.

Capafons, A., Alarcón, A., Cabañas, S., \& Espejo, B. (2003). Análisis factorial exploratorio y propiedades psicométricas del cuestionario de creencias y actitudes hacia la hipnosis-cliente. Psicothema, 15, 143-147. podría generar iatrogenia. Estos resultados son destacables teniendo en cuenta que son estudiantes de primeros cursos, aunque la mayoría sean de Psicología.

También se podría investigar con muestras españolas las relaciones de EVACH-C con otras escalas similares, que ahora empiezan a ser reanalizadas con metodología factorial más avanzada (Milling, 2012), así como estudiar las relaciones de la EVACH-C con escalas de evaluación de sugestionabilidad hipnótica, tal como ya ha hecho Green (2012) con muestra de EE.UU.

En la medida en que las actitudes y creencias hacia la hipnosis predicen el resultado terapéutico cuando se inserta la hipnosis en una intervención, es perentorio realizar investigaciones que estudien la capacidad predictiva de la EVACH-C de los resultados, y compararla con otras variables como sugestionabilidad hipnótica, motivación, etc.

También se deberían buscar combinaciones de esas variables para encontrar conjuntos de predictores que puedan ayudar a dirimir la conveniencia, o no, de incluir la hipnosis dentro de una intervención. De este modo, se ayudaría al profesional a seleccionar los instrumentos que le sugieran qué pacientes presentan una mayor probabilidad de beneficiarse de los tratamientos que incluyen la hipnosis, quiénes necesitan información más precisa sobre la hipnosis para no desarrollar iatrogenia, incluso si deben recibir una mínima intervención para incrementar su sugestionabilidad hipnótica y con ello mejorar el rendimiento del tratamiento (Capafons \& Mendoza, 2010; Gfeller \& Gorassini, 2010).

Finalmente, deberían replicarse las investigaciones que se realizaron con la versión anterior de la EVACH-C (Capafons et al., 2005; Capafons et al., 2006) y con la EVACH-T (Martín et al., 2010; Mendoza, Capafons \& Espejo, 2009) para observar si esta variación detecta cambios en actitudes y creencias una vez se ha intervenido sobre ellas, lo que aportaría información valiosa para la validez de constructo de la escala.
Capafons, A., Cabañas, S., Alarcón, A. Espejo, B., Mendoza, Mª E., Chaves, J. F., \& Monje, A. (2005). Effects of different types of preparatory information on attitudes toward hypnosis. Contemporary Hypnosis, 22, 6776.

Capafons, A., Cabañas, S., Espejo, B., \& Cardeña, E. (2004). Confirmatory factor analysis of the Valencia scale on attitudes and beliefs toward hypnosis: An international study. International Journal of Clinical and Experimental Hypnosis, 52, 413-433.

Capafons, A., Espejo, B, \& Cabañas, S. (2005). Creencias que pueden impedir que la hipnosis sea una técnica útil para la salud: un estudio exploratorio con muestra cubana. Revista del Hospital Psiquiátrico de la Habana, 2, Recuperado http://www.revistahph.sld.cu/hph0205/hph020105.htm

Capafons, A., Espejo, B., \& Mendoza, M. (2008). Confirmatory factor analysis of the Valencia scale on attitudes and beliefs toward hypnosis, therapist version. International Journal of Clinical and Experimental Hypnosis, 56, 281-294.

Capafons, A., Flores, D., Suárez-Rodríguez, J. M., \& Lopes-Pires, C. (2010, julio): análisis factorial confirmatorio con muestra portuguesa de la escala de Valencia de actitudes y creencias hacia la hipnosis-cliente. En A. Capafons (Chair): Avances en hipnosis experimental y aplicada. Simpo- 
sio celebrado en el VII Congreso Iberoamericano de Psicología 2010, Oviedo (España).

Capafons, A., Lamas, J. R., \& Lopes-Pires, C. (2008). Hipnosis. En F. J. Labrador (Ed.), Técnicas de modificación de conducta (pp. 593-614). Madrid: Pirámide.

Capafons, A., \& Mazzoni, G. (2005). ¿Es lo peligroso de la hipnosis el hipnoterapeuta?: hipnosis y falsos recuerdos. Papeles del Psicólogo, 25, 27-38.

Capafons, A., \& Mendoza, E. (2010). Waking hypnosis in clínical practice. En S. J. Lynn, J. W. Rhue, \& I. Kirsch (Eds.), Handbook of clinical bypnosis ( $2^{\mathrm{a}}$ ed.) (pp. 293-317). Washington, D.C.: American Psychological Association.

Capafons, A., Mendoza, Mª E., Espejo, B., Green, J. P., Lopes-Pires, C., Selma, Ma L., ... Carvallho, C. (2008). Attitudes and beliefs about hypnosis: A multicultural study. Contemporary Hypnosis, 25, 141-155.

Capafons, A., Morales, C., Espejo, B. \& Cabañas, S. (2006). Análisis factorial exploratorio y propiedades psicométricas de la escala de Valencia de actitudes y creencias hacia la hipnosis, versión terapeuta. Psicothema, 18, 810-815.

Capafons, A., Selma, M. L., Cabañas, S., Espejo, B., Alarcón, A., Mendoza, M E., \& Nitkin-Kaner, Y. (2006). Change of attitudes toward hypnosis: Effects of cognitive-behavioral and trance explanations in a setting of heterohypnosis. Australian Journal of Clinical and Experimental Hypnosis, 34, 119-134.

Carvalho, C., Capafons, A., Kirsch, I., Espejo, B., Mazzoni, G., \& Leal, I. (2007). Factorial analysis and psychometric properties of the revised Valencia Scale of Attitudes and Beliefs Toward Hypnosis-Client Version. Contemporary Hypnosis, 24, 76-85.

Crawford, H. J., Knebel, T., Vendemia, J. M. C., Horton, J. E., \& Lamas, J. R. (1999). La naturaleza de la analgesia hipnótica: bases y evidencias neurofisiológicas. Anales de Psicología, 15, 133-146.

Cronbach, L. J. (1951). Coefficient Alpha and the internal structure of tests. Psychometrika, 16, 297-334.

Echterling, L. G., \& Whalen, J. (1995). Stage hypnosis and public lecture effects on attitudes and beliefs regarding hypnosis. American Journal of Clinical Hypnosis, 38, 13-21.

Eimer, B. N., \& Freeman, A. (1998). Pain management psychotherapy: A practical guide. NewYork, NY: John Wiley \& Sons.

Gfeller, J. D., \& Gorassini, D. R. (2010). Enhancing hypnotizability and treatment response. En S.J. Lynn, J.W. Rhue, \& I. Kirsch (Eds.), Handbook of clinical hypnosis (2 ed.) (pp. 339-355). Washington, D.C.: American Psychological Association.

Green, J. (2012). The Valencia scale of attitudes and beliefs toward hypnosis-client version and hypnotizability. International Journal of Clinical and Experimental Hypnosis, 60, 229-240.

Green, J. P., Houts C.R., \& Capafons, A. (2012). Attitudes about hypnosis: Factor analyzing the VSABTH-C with an American sample. American Journal of Clinical Hypnosis, 54, 167-178.

Hawkins, R., \& Bartsch, J. (2000). The effects of an educational lecture about hypnosis. Australian Journal of Clinical and Experimental Hypnosis, 28, 82-99.

Hair, J. F., Black, W. C., Babib, B. J., \& Anderson, R. E. (2010). Multivariate data Analysis ( $7^{a}$ ed.). Upper Saddle River, NJ: Prentice Hall.

Hooper, D., Coughlan, J., Mullen, M. (2008). Structural Equation Modelling: Guidelines for determining model fit. Electronic Journal of Business Research Methods, 6, 53-60.

Jensen, M. J. (2011). Hypnosis for chronic pain management. Therapist guide. New York, NY. Oxford University Press.

Jöreskog, K. G., Sörbom, D., Du Toit, S., \& Du Toit, M. (1999). LISREL 8: New statistical features. Chicago, IL: Scientific Software International, Inc.

Keller, R. F. (1996). Hypnosis belief survey. Psychological Hypnosis, 5, 8-9.

Kline, R. B. (2005). Principles and practice of structural equation modeling ( $\left.2^{\mathrm{a}} \mathrm{ed}.\right)$. New York, NY: Guilford Press.
Martín, M., Capafons, A., Espejo, B., Mendoza, M. E., Guerra, M., Enríquez-Santos, J.A., ... Sosa, C. D. (2010). Impact of a lecture about empirical bases of hypnosis on beliefs and attitudes toward hypnosis among Cuban health professionals. International Journal of Clinical and Experimental Hypnosis, 58, 476-496.

McConkey, K. M. (1986). Opinions about hypnosis and self-hypnosis before and after hypnotic testing. International Journal of Clinical and Experimental Hypnosis, 34, 311-319.

McConkey, K. M., \& Jupp, J. J. (1985-1986). A survey of opinions about hypnosis. British Journal of Experimental Hypnosis, 3, 87-93.

Mendoza, M ${ }^{a}$ E., \& Capafons, A. (2009). Eficacia de la hipnosis clínica: resumen de su evidencia empírica. Papeles del Psicólogo, 30, 98-116.

Mendoza, Ma E., Capafons, A., \& Espejo, B. (2009): Impact of reading a scientific journal issue about hypnosis on the beliefs and attitudes towards hypnosis among psychologists. Psychology, Society, \& Education, 1, 25-37.

Mendoza, M ${ }^{a}$ E., Capafons, A., Espejo, B., \& Montalvo, D. (2009). Creencias y actitudes hacia la hipnosis de los psicólogos españoles afiliados al Colegio Oficial de Psicólogos. Psicothema, 21, 465-470.

Mendoza, $\mathrm{M}^{\mathrm{a}}$ E., Capafons, A., \& Flores, D. (2009). Instrumentos psicométricos para la evaluación de las creencias y actitudes hacia la hipnosis. En C. M. Lopes Pires \& E. Santos (Eds.), Hipnose clínica: Fundamentos e aplicações em psicologia e saúde (pp. 59-75). Viseu, Portugal: Psicossoma.

Milling, L. S. (2012). The Spanos attitudes toward hypnosis questionnaire: Psychometric characteristics and normative data. American Journal of Clinical Hypnosis, 54, 202-212.

Nickisson, J. W. (1997). Hypnosis: attitudes, knowledge and prior experience among psychology and nursing students. Tesis publicada, University of Northern Colorado, CO., EE.UU.

Orgilés, M., Méndez, F. X., \& Espada, J. P. (2009). Procedimientos psicológicos para el afrontamiento del dolor en niños con cáncer. Psicooncología, $6,343-356$.

Palanca Sánchez, I. (Dir.), Puig-Riera de Conías, M. M. (Coord.), ElolaSomoza, J. (Dir), Bernal-Sobrino, J. L. (Comit. Redac.), PaniaguaCaparrós J. L. (Comit. Redac.), \& Grupo de Expertos (2011). Unidad de tratamiento de dolor: estándares y recomendaciones de calidad y seguridad. Madrid: Ministerio de Sanidad, Política Social e Igualdad. Recuperado de http://www.msc.es/organizacion/sns/planCalidadSNS/docs/EERR/ Unidad de tratamiento_del_dolor.pdf

Raykov, T. (2001). Testing multivariable covariance structure and means hypotheses via structural equation modeling. Structural Equation Modeling, 2, 224-257.

Raykov, T. (2004). Point and interval estimation of reliability for multiplecomponent measuring instruments via linear constraint covariance structure modeling. Structural Equation Modeling, 11, 342-356.

Schoenberger, N. E., Kirsch, I., Gearan, P., Montgomery, G., \& Pastyrnak, S. L. (1997). Hypnotic enhancement of a cognitive behavioral treatment for public speaking anxiety. Behavior Therapy, 28, 127-140.

Satorra, A., \& Bentler, P. M. (1994). Corrections to test statistics and standard errors in covariance structure analysis. En A. van Eye \& C. C. Clogg (Eds.), Latent variable analysis in developmental research (pp. 399-419). Thousand Oaks, CA: Sage Publications.

Sliwinskiand, J. G., \& Elkins, R. (2013). Enhancing placebo effects: Insights from social psychology. American Journal of Clinical Hypnosis, 55, 236-248.

Spanos, N. P., Brett, P. J., Menary, E. P., \& Cross, W. P. (1987). A measure of attitudes toward hypnosis: Relationships with absorption and hypnotic susceptibility. American Journal of Clinical Hypnosis, 30, 139-150.

Truyols-Taberner, M M., Pérez-Pareja, J., Palmer-Pol, A., \& Sesé-Abad, A. (2008). Aspectos psicológicos relevantes en el estudio y tratamiento del dolor crónico. Clínica y Salud, 19, 295-320.

(Articulo recibido: 18/03/2013; revisado: 28/07/2013; aceptado: 29/10/2013) 


\section{Apéndice.}

EVACH-C

Todos los datos personales recogidos en esta escala están sometidos al secreto profesional, por lo que no podrán hacerse públicos sin su autorización expresa

\section{GRACIAS POR SU COLABORACIÓN}

NOMBRE

APELLIDOS

PAIS

TELÉFONO (opcional)

CIUDAD

EDAD

FECHA.

1. Indique su Estado Civil (rodee lo que proceda):
1. Soltero/a
2. Casado/a
3. Viudo/a
4. Separado/a o divorciado/a

2. Indique su sexo:

1. Hombre

2. Mujer

3. ¿Trabaja actualmente?

1. Sí

2. No

4. En caso de que sí, indique su trabajo

5. ¿Ha sido hipnotizado alguna vez? $\quad 1$ Sí 2 . No

6. En caso de que sí, ¿por quién o quiénes? (marcar todas las opciones que procedan)

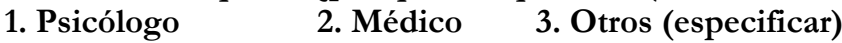

7. ¿Ha recibido alguna información acerca de la hipnosis?

1. Sí 2. No

8. En caso de que sí, indique la fuente de la que ha obtenido dicha información (marcar todas las opciones que procedan):

\section{Universidad}

5. Televisión

2. Cursos

3. Lecturas científicas

4. Otro tipo de lecturas

9. Si además es estudiante:

1. Indique la titulación que está cursando

2. Indique el curso

3. Indique la Universidad donde cursa sus estudios

4. Indique el nombre de la población y país en la que cursa sus estudios

10. Indique otras titulaciones que posea

A continuación encontrará algunas cuestiones que nos ayudarán a conocer su opinión sobre la hipnosis. No hace falta que haya pasado por la experiencia sobre la que se le pregunta, sólo que indique lo que considera que podría ocurrir en tales situaciones. Por favor, indique su grado de acuerdo o desacuerdo con las afirmaciones que se le presentan más abajo, $\underline{\text { ro- }}$ deando el número que mejor refleje su opinión, según la siguiente escala:

1. Completamente en desacuerdo

2. Bastante en desacuerdo

3. En desacuerdo

4. De acuerdo

5. Bastante de acuerdo

6. Completamente de acuerdo

RECUERDE QUE NO HAY RESPUESTAS BUENAS O MALAS, ÚNICAMENTE SE TRATA DE CONOCER SU OPINIÓN

1. La hipnosis puede ser de gran ayuda para otros

2. La hipnosis implica un esfuerzo de cooperación entre el hipnotizador y el cliente

3. Se necesita estar en un trance hipnótico para conseguir las metas de la intervención

4. La hipnosis me da miedo

5. Bajo hipnosis se consiguen cosas sin ningún esfuerzo por parte de la persona

6. La hipnosis puede ser una solución mágica a los problemas de la persona hipnotizada

7. Creo que bajo hipnosis la persona es un autómata a merced del hipnotizador 
8. La hipnosis requiere esfuerzo por parte de la persona hipnotizada

123456

9. La hipnosis es todo lo que se necesitaría para tratar la mayoría de problemas 123456

10. La hipnosis puede ser de gran ayuda para mejorar los efectos de los tratamientos a los que se adjunta

11. La persona hipnotizada es pasiva

12. La hipnosis es un complemento o herramienta para ayudar a las terapias psicológicas 123456 CONTINÚE, POR FAVOR

\section{Completamente en desacuerdo \\ 2. Bastante en desacuerdo \\ 3. En desacuerdo \\ 4. De acuerdo \\ 5. Bastante de acuerdo \\ 6. Completamente de acuerdo}

13. Para hipnotizar a alguien es necesaria su colaboración 123456

14. La persona hipnotizada puede "salir" de hipnosis cuando lo desee

123456

15. Bajo hipnosis la persona conserva su voluntad para hacer lo que quiera

123456

16. La hipnosis es una técnica segura, con pocos riesgos

123456

17. La hipnosis fomenta la capacidad de auto-control 123456

18. Tengo miedo de quedarme "enganchado" en un trance hipnótico

123456

19. Creo que bajo hipnosis se puede llegar a perder el control sobre uno mismo

123456

20. Creo que la hipnosis puede resultar peligrosa

123456

21. Todo lo que ocurre bajo hipnosis lo provoca la persona hipnotizada

123456

22. Bajo hipnosis pueden obligar a la persona a hacer cosas que no desee

123456

23. La hipnosis es un facilitador de los resultados terapéuticos

123456

24. Si la persona está en contra de una sugestión puede ignorarla completamente

123456

25. La persona hipnotizada mantiene el control sobre sí misma

123456

26. Me gustaría ser hipnotizado

123456

27. Me dejaría hipnotizar si se diera el caso

123456

28. Me gustaría ser muy hipnotizable

123456

29. Se aprende más deprisa bajo hipnosis

123456

30. Lo que se recuerda bajo hipnosis es la verdad .......................................................................... 123456

31. Es imposible mentir bajo hipnosis, aunque la persona hipnotizada lo desee .......................... 123456

32. Una forma de confirmar que un suceso ocurrió es que la persona lo recuerde bajo hipnosis 123456

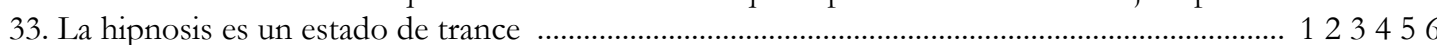

34. La hipnosis se desarrolla al margen de la investigación científica ............................................. 123456

35. En general, algunas de las características fundamentales de las personas muy hipnotizables serían: credulidad, ignorancia y dependencia psicológica .............................................................. 123456

36. La persona hipnotizada se encuentra disociada ........................................................................ 123456

37. La hipnosis es un complemento o herramienta para ayudar a las terapias médicas.................. 123456

GRACIAS POR SU COLABORACIÓN 